\title{
Workshop on Gamification for Information Retrieval (GamifIR'14)
}

\author{
Frank Hopfgartner ${ }^{1}$, Gabriella Kazai $^{2}$, Udo Kruschwitz ${ }^{3}$, and Michael Meder ${ }^{1}$ \\ 1 Technische Universität Berlin, Berlin, Germany \\ \{firstname.lastname\}@tu-berlin.de \\ ${ }_{2}$ Microsoft Research, Cambridge, UK \\ v-gabkaz@microsoft.com \\ 3 University of Essex, Colchester, UK \\ udo@essex.ac.uk
}

\begin{abstract}
Gamification is the application of game mechanics, such as leader boards, badges or achievement points, in non-gaming environments with the aim to increase user engagement, data quality or cost effectiveness. A core aspect of gamification solutions is to infuse intrinsic motivations to participate by leveraging people's natural desires for achievement and competition. While gamification, on the one hand, is emerging as the next big thing in industry, e.g., an effective way to generate business, on the other hand, it is also becoming a major research area. However, its adoption in Information Retrieval (IR) is still in its infancy, despite the wide ranging IR tasks that may benefit from gamification techniques. These include the manual annotation of documents for IR evaluation, the participation in user studies to study interactive IR challenges, or the shift from single-user search to social search, just to mention a few.

This context provided the motivation to organise the GamifIR'14 workshop at ECIR.
\end{abstract}

\section{Motivation}

Many research challenges in the field of IR rely on tedious manual labour. For example, manual feedback is required to assess the relevance of documents to a given search task, to annotate documents or to evaluate interactive IR approaches. A recent trend to perform these tasks is the use of crowdsourcing techniques, i.e., relying on human computation and the "wisdom of the crowd" [1]. Although research indicates that such techniques can be useful, they fail when motivated users are required to perform a task for reasons other than just being paid per click or paid per minute [2].

A promising approach to increase user motivation can be achieved by employing gamification methods, i.e., game mechanics and principles such as leaderboards, points for certain activities or achievement badges are applied in nongaming environments to reach specific goals (e.g. [3-6]).

Recently, gamification has drawn the attention of researchers from various fields, leading to workshops (e.g., [7-9]) and the eventual introduction of a new 
conference on gamification [10]. At the same time, the IR community focused on the use of crowdsourcing for relevance evaluation [11] and at searching for fun (e.g., [12]), two topics which can benefit from the use of gamification principles.

This workshop focused on the challenges and opportunities that gamification can present for the IR community. The workshop aimed to bring together researchers and practitioners from a wide range of areas including game design, information retrieval, human-computer interaction, computer games, and natural language processing. Detailed information about the workshop can be found on the workshop website at http://gamifir2014.dai-labor.de/.

\section{Workshop Scope}

The call for papers solicited submissions of position papers as well as novel research papers and demos addressing problems related to gamification in IR including topics such as:

- Gamification approaches in a variety of information-seeking contexts

- User engagement and motivational factors of gamification

- Player types, contests, cooperative games

- Challenges and opportunities of applying gamification in IR

- Gamification design and game mechanics

- Game-based work and crowdsourcing

- Applications and prototypes

- Evaluation of gamification techniques

\section{Keynote}

We were very pleased that Prof. Richard Bartle of the University of Essex (United Kingdom) agreed to provide a keynote at GamifIR'14. Bartle is one of the key researchers in the field of game design. His research lay the ground for the success of massively multiplayer online games. The gamification research community knows him best for his Player Types model [13] in which he defines different types of players that can be distinguished based on their individual behaviour.

\section{Acknowledgements}

We acknowledge the efforts of the members of the programme committee, namely:

- Omar Alonso (Microsoft Research, USA),

- Leif Azzopardi (University of Glasgow, United Kingdom),

- Yoram Bachrach (Microsoft Research, United Kingdom),

- Regina Bernhaupt (Université Paul Sabatier, France),

- Jon Chamberlain (University of Essex, United Kingdom),

- Edwin Chen (YouTube/Google, USA),

- Sebastian Deterding (Rochester Institute of Technology, USA), 
- Carsten Eickhoff (ETH Zürich, Switzerland),

- Rosta Farzan (University of Pittsburgh, USA),

- Christopher G. Harris (The University Of Iowa, USA),

- Shaili Jain (Microsoft, USA),

- Hideo Joho (University of Tsukuba, Japan),

- Mounia Lalmas (Yahoo! Labs, Spain),

- Edith Law (Harvard University, USA),

- David Parkes (Harvard University, USA),

- Massimo Poesio (University of Essex, United Kingdom),

- Falk Scholer (RMIT, Australia),

- Craig Stewart (Coventry University, United Kingdom),

- Elaine Toms (University of Sheffield, United Kingdom)

- Arjen de Vries (CWI, The Netherlands),

- Albert Weichselbraun (University of Applied Sciences Chur, Switzerland),

- Lincoln C. Wood (Auckland University of Technology, New Zealand).

\section{References}

1. Alonso, O., Mizzaro, S.: Using crowdsourcing for TREC relevance assessment. Inf. Process. Manage. 48(6) (2012) 1053-1066

2. Kazai, G., Kamps, J., Milic-Frayling, N.: An analysis of human factors and label accuracy in crowdsourcing relevance judgments. Inf. Retr. 16(2) (2013) 138-178

3. Eickhoff, C., Harris, C.G., de Vries, A.P., Srinivasan, P.: Quality through flow and immersion: gamifying crowdsourced relevance assessments. In: SIGIR. (2012) $871-880$

4. Poesio, M., Chamberlain, J., Kruschwitz, U., Robaldo, L., Ducceschi, L.: Phrase Detectives : Utilizing Collective Intelligence for Internet-Scale. ACM Trans. Interact. Intell. Syst. 3(1) (2013)

5. Meder, M., Plumbaum, T., Hopfgartner, F.: Perceived and Actual Role of Gamification Principles. In: UCC'13: Proceedings of the IEEE/ACM 6th International Conference on Utility and Cloud Computing, IEEE (12 2013) 488-493

6. Meder, M., Plumbaum, T., Hopfgartner, F.: DAIKnow: A Gamified Enterprise Bookmarking System. In: ECIR'14: Proceedings of the 36th European Conference on Information Retrieval, Springer Verlag (04 2014)

7. Deterding, S., Sicart, M., Nacke, L., O'Hara, K., Dixon, D.: Gamification. using game-design elements in non-gaming contexts. In: CHI '11 Extended Abstracts on Human Factors in Computing Systems. CHI EA '11, New York, NY, USA, ACM (2011) 2425-2428

8. Deterding, S., Björk, S.L., Nacke, L.E., Dixon, D., Lawley, E.: Designing gamification: Creating gameful and playful experiences. In: CHI '13 Extended Abstracts on Human Factors in Computing Systems. CHI EA '13, ACM (2013) 3263-3266

9. Springer, T., Herzig, P., eds.: Proceedings of the 2013 IEEE/ACM 6th International Conference on Utility and Cloud Computing - International Workshop on Gamification in the Cloud. CGCloud'13 (2013)

10. Nacke, L., Harrigan, K., Randall, N., eds.: Proceedings of the First International Conference on Gameful Design, Research, and Applications. Gamification'13 (2013) 
11. Alonso, O., Lease, M.: Crowdsourcing for information retrieval: Principles, methods, and applications. In: Proceedings of the 34th International ACM SIGIR Conference on Research and Development in Information Retrieval. SIGIR '11, New York, NY, USA, ACM (2011) 1299-1300

12. Elsweiler, D., Wilson, M.L., Harvey, M., eds.: Proceedings of the "Searching 4 Fun!" workshop. Volume 836 of CEUR. (2012)

13. Bartle, R.: Hearts, clubs, diamonds, spades: Players who suit MUDs. The Journal of Virtual Environments 1(1) (1996) available online. 